\title{
Selective Spectral Modulation of Strongly Coupled Spins with an Echo Top Refocusing Pulse in PRESS Sequences
}

\author{
Giulio Gambarota ${ }^{1,2,3}$, Arnaud Bondon ${ }^{3,4}$, Marie Le Floch ${ }^{5,6}$, Robert V. Mulkern ${ }^{7}$, Hervé \\ Saint-Jalmes ${ }^{1,2,3}$
}

${ }^{1}$ INSERM, UMR 1099, Rennes, F-35000, France

${ }^{2}$ Université de Rennes 1, LTSI, Rennes, F-35000, France

${ }^{3}$ PRISM - Biosit, Biogenouest, Rennes, F-35000, France

${ }^{4}$ Université de Rennes 1, SIM, UMR CNRS 6290, Rennes, F-35000, France

${ }^{5}$ CNRS - UMR "Sciences Chimiques de Rennes", Université de Rennes 1, Verres et Céramiques, Rennes, F-35000, France

${ }^{6}$ Université Européenne de Bretagne, Rennes, F-35000, France

${ }^{7}$ Department of Radiology, Children's Hospital, Harvard Medical School, Boston, MA, USA.

Running Head: Selective Modulation of Strongly Coupled Spins.

Keywords: strongly coupled spin systems; double spin echo; refocusing pulses; coherence transfer; density matrix theory; PRESS; citrate.

Corresponding author:

Giulio Gambarota, Ph.D.

LTSI, Université de Rennes 1

2, avenue du Professeur Léon Bernard

35043 Rennes, France

Tel: 0223234849

email: gambarota@gmail.com 


\begin{abstract}
The double spin echo is the basis of the point resolved spectroscopy (PRESS) sequence. In this study we sought to investigate the effects of a broadband $180^{\circ}$ pulse -incorporated in the PRESS sequence at the location of the first echo (gPRESS)- on the citrate resonances, chosen as a model of strongly coupled spin system. A significant signal modulation generated by the additional $180^{\circ}$ pulse was predicted with simulations and observed experimentally in the citrate resonances. No effects were observed on the singlet resonance of glycine and the weakly coupled resonances of lactate. The phenomenon observed in gPRESS was attributed to the off-diagonal Hamiltonian elements responsible for a coherence transfer occurring throughout the evolution periods. The results of this study show that it is necessary to assess the effects of broadband $180^{\circ}$ pulses on strongly coupled spin systems, since these pulses can selectively modify the spectral shape of strongly coupled resonances.
\end{abstract}




\section{Introduction}

The double spin echo is the basis of the point resolved spectroscopy (PRESS) localization sequence, one of the most commonly used ${ }^{1} \mathrm{H}$ MRS sequences in clinical settings [1]. A number of modifications to the standard PRESS scheme have been introduced to improve in vivo metabolite detection [2]. An early modification was the so-called 'perfect echo' sequence in which the J-modulation of weakly coupled resonances is partially refocused by inserting a $90^{\circ}$ pulse between the refocusing pulses, at the location of the first echo. This idea was proposed independently by Takegoshi et al. [3] and van Zijl et al. [4] more than two decades ago with the extension to strongly coupled spin systems investigated by Mulkern et al. [5] a few years later.

Many modifications to PRESS ensued. Various quantum coherence filtering schemes have been incorporated with the aim of improving in vivo detection of coupled spin systems such as GABA, glutamate and glutathione [6-9]. Other modifications to PRESS have been designed to minimize the deleterious effects of chemical shift displacement that are exacerbated at higher fields. In this respect, one relevant example is the 'four-pulse PRESS' (or 'PRESS +4 ') [10-12], where a non-selective $180^{\circ}$ pulse has been added at the location of the first echo. It was shown that the additional non-selective $180^{\circ}$ pulse significantly reduced the signal loss from the chemical shift displacements in the weakly coupled spin resonances of lactate and GABA [10-12]. However, no investigations were performed to determine the effects of the non-selective $180^{\circ}$ pulse on strongly coupled spin resonances. As a matter of fact, $180^{\circ}$ pulses can modify the spectral shape and signal intensity of strongly coupled spin systems [13-16]. For instance, it has been shown that the response of strongly coupled resonances displays a significant dependence on the PRESS interpulse delays, at constant TE, with large signal modulations up to a complete inversion of the spectral lineshape of citrate at $3 \mathrm{~T}$ and no effects on weakly coupled spin resonances [13].

Motivated by these novel modifications available for the PRESS localization scheme [10-12], we sought to investigate the effects on a strongly coupled two-spin system of an additional $180^{\circ}$ refocusing pulse in the PRESS sequence. This pulse sequence is here referred to as gPRESS. The primary hypothesis is that when placed between the refocusing pulses, at the location of the first echo, a non-selective (i.e., broadband) $180^{\circ}$ pulse would have significant effects on the signal of strongly coupled resonances with no effect on uncoupled and weakly coupled spins. 


\section{Materials and Methods}

In previous studies, it has been shown that the signal behaviour of coupled spins in PRESS can be well described with a double spin echo [4-6, 13-15, 17-20]. Thus, in all simulations presented here hard pulses and no spatial localization were assumed. The effects of an additional $180^{\circ}$ pulse on citrate proton resonances were first investigated with simulations based on the quantum-mechanical density matrix theory [21-23]. Specifically, a $180^{\circ}$ pulse was placed at the location of the first echo of a double spin echo sequence with the following timings: $90^{\circ}{ }_{\mathrm{x}}-\left[\mathrm{TE}_{1} / 2\right]-180^{\circ}{ }_{\mathrm{y}}-\left[\mathrm{TE}_{1} / 2\right]-180^{\circ} \mathrm{y}-\left[\mathrm{TE}_{2} / 2\right]-180^{\circ}{ }_{\mathrm{y}}-\left[\mathrm{TE}_{2} / 2\right]-$ Acq, where $\mathrm{TE}=\mathrm{TE}_{1}+\mathrm{TE}_{2}$. Simulations were performed on the citrate proton resonances, which can be modelled as a two-spin system. At typical magnetic field strengths of clinical scanners, it is well known that citrate (chemical shift difference $\Delta=0.146 \mathrm{ppm}$, J-coupling constant of 15 $\mathrm{Hz}$ ) can be considered as a strongly coupled two-spin (AB) system [17-20]. For instance, at 3 $\mathrm{T}, \Delta$ is equal to $18 \mathrm{~Hz}$, which is close to the J-coupling constant. At the field strength $(11.7 \mathrm{~T})$ of the spectrometer employed in the current study, the ratio of the J-coupling constant to chemical shift difference is about 0.2 , which still falls under the strong coupling regime. Thus, in this study citrate was chosen as a model of an AB spin system.

The density matrix operator $\sigma(\mathrm{t})$ for gPRESS sequence can be written as: $\sigma(t)=g^{-} F_{z} g^{+}$, where

$$
g^{-}=e^{-i t H} e^{-i\left(T E_{2} / 2\right) H} e^{-i \pi F_{y}} e^{-i\left(T E_{2} / 2\right) H} e^{-i \pi F_{y}} e^{-i\left(T E_{1} / 2\right) H} e^{-i \pi F_{y}} e^{-i\left(T E_{1} / 2\right) H} e^{-i(\pi / 2) F_{x}}
$$

and

$$
g^{+}=e^{i(\pi / 2) F_{x}} e^{i\left(T E_{1} / 2\right) H} e^{i \pi F_{y}} e^{i\left(T E_{1} / 2\right) H} e^{i \pi F_{y}} e^{i\left(T E_{2} / 2\right) H} e^{i \pi F_{y}} e^{i\left(T E_{2} / 2\right) H} e^{i t H}
$$

Each exponential term in $\mathrm{g}^{-}$and $\mathrm{g}^{+}$is a $4 \mathrm{x} 4$ complex-valued matrix, where $\mathrm{H}$ is the Hamiltonian of the $\mathrm{AB}$ spin system and $\mathrm{F}_{\mathrm{z}}, \mathrm{F}_{\mathrm{x}}$, and $\mathrm{F}_{\mathrm{y}}$ are the $\mathrm{z}, \mathrm{x}$, and $\mathrm{y}$ magnetic moment operators, respectively. All the angular momentum and rotation operators were expressed in the spin-product basis [13]. The transverse magnetization at time $t$ was found by calculating the trace of the product of the density matrix operator with the transverse-magnetization operator $\mathrm{F}_{+}$, with $\mathrm{F}_{+}=\mathrm{F}_{\mathrm{x}}+\mathrm{i} \mathrm{F}_{\mathrm{y}}$. To investigate the effects of the additional $180^{\circ}$ pulse, plots of the signal intensity of citrate as a function of the interpulse delays in PRESS and gPRESS were generated. Increments of $1 \mathrm{~ms}$ were used for the interpulse delays. The signal intensity is defined here as the integrated peak area, i.e., the integration over the frequency domain of the citrate signal. For computational simplicity, the signal intensity was obtained from the value 
of the first point of the FID, rather than from integration of the entire spectrum [13]. Furthermore, an analytic solution for the time dependent signal was derived using the density matrix formalism (see Table 1, Table 2 and appendix for details), assuming hard pulses and without taking into account the effects of localization gradients. In general, to assess the performance of gPRESS in clinical settings, more detailed simulations/calculations with the inclusion of soft pulses and localization gradients would be necessary.

Experiments were performed on a Bruker Avance $500 \mathrm{MHz}$ spectrometer equipped with a 5 $\mathrm{mm}$ broadband observe probe. Similarly to the simulations, experiments were conducted with hard pulses. Spectra were acquired on a phantom containing $25 \mathrm{mM}$ citrate, $20 \mathrm{mM}$ glycine and $35 \mathrm{mM}$ lactate in deuterated water. Glycine and lactate were chosen as models of singlet and weakly coupled spin resonances, respectively. The solution was buffered by addition of sodium phosphate $\mathrm{pH} 6.8$ at a final concentration of $50 \mathrm{mM}$. A spectral width of $7 \mathrm{kHz}$ was used with $32 \mathrm{~K}$ points corresponding to an acquisition time of $2.34 \mathrm{~s}$. The duration of the $90^{\circ}$ hard pulse was $11.7 \mu \mathrm{s}$. A presaturation of the residual water signal was applied during a recovery delay of $3 \mathrm{~s}$. Apodization by a line broadening of $5 \mathrm{~Hz}$ was performed before Fourier transform and chemical shifts were referenced to internal trimethylsilyl propionic acid (TSP). 


\section{Results}

Simulations of the signal intensity of the citrate resonances at $11.7 \mathrm{~T}$ following double spin echo excitation with (gPRESS) and without (PRESS) the additional $180^{\circ}$ placed at the location of the first echo were performed (Figure 1). To highlight the effects of the $180^{\circ}$ pulse, the difference between the signal intensity of PRESS and gPRESS is shown in Figure 1. The additional $180^{\circ}$ pulse generated changes in signal intensity up to $50 \%$, for TE in the range of $60-80 \mathrm{~ms}, 120-140 \mathrm{~ms}$ and $180-200 \mathrm{~ms}$ (Figure 1). Simulations of the citrate resonances at $\mathrm{TE}=132 \mathrm{~ms}\left(\mathrm{TE}_{1}=20 \mathrm{~ms}\right)$ showed the large differences in spectral shape that were generated by the $180^{\circ}$ pulse (Figure 2). In gPRESS, the signal intensity of the inner lines of the citrate multiplet completely disappeared at this sequence timing. A similar signal behavior of the citrate resonances was observed experimentally (Figure 3). It is also noticeable that the $180^{\circ}$ pulse had no effect on the singlet resonance of glycine and on the weakly coupled resonances of lactate. To illustrate a potential application of gPRESS, simulations of the spectral lineshape of the citrate resonances at $3 \mathrm{~T}$ under gPRESS and PRESS excitation are depicted in Figure 4. The spectrum from the PRESS sequence at TE = $145 \mathrm{~ms}$-a commonly used TE for citrate at 3T [24]- is shown for comparison. Note that the earlier echo time achievable with gPRESS and with a favorable lineshape should help ameliorate T2 decay signal losses at 3T compared to the longer TE of the PRESS sequence. Furthermore, in Figure 4 the citrate signal for PRESS at TE $=67 \mathrm{~ms}$ and gPRESS at $145 \mathrm{~ms}$ is also shown, for comparison purposes. It should be noted that the citrate signal for PRESS at $\mathrm{TE}=67 \mathrm{~ms}$ is not advantageous due to its inverted lines that might generate signal cancellation with neighboring peaks in inhomogeneous voxels, as well as difficulties in spectral processing [25]. Citrate spectra at $11.7 \mathrm{~T}$ for PRESS and gPRESS obtained by the analytic solution $\left(\mathrm{TE}=132 \mathrm{~ms}\right.$ and $\mathrm{TE}_{1}=20 \mathrm{~ms}$, Figure 5) are in excellent agreement with the spectra obtained from density matrix simulations (Figure 2) and with experimental data (Figure 3). 


\section{Discussion and Conclusions}

Refocusing $180^{\circ}$ pulses and strongly coupled spin systems are ubiquitous in NMR spectroscopy. Here we show that a broadband $180^{\circ}$ pulse inserted at the location of the first echo in a PRESS sequence has significant effects on citrate resonances, which are chosen here as a model of strongly coupled two-spin system. The effect is such that, at certain values of interpulse delays, the additional $180^{\circ}$ pulse completely suppresses the inner lines of the citrate multiplet.

The ability to manipulate the response of coupled spin resonances has been one of the main topics in the field of MR spectroscopy though surprisingly, only a few studies have investigated the effects of $180^{\circ}$ pulse on strongly coupled spin systems [13-16]. It is well known that broadband $180^{\circ}$ pulses refocus the chemical shift evolution without refocusing the evolution due to the homonuclear scalar interactions (J-coupling) between spins. The phenomenon observed in this study can be analysed within the framework of the density matrix theory. Its origin can be attributed to the off-diagonal elements of the Hamiltonian in the Zeeman basis. These terms are in fact responsible for a coherence transfer occurring throughout the evolution periods in the absence of RF pulses. It should be stressed that, for weakly coupled spins, the off-diagonal Hamiltonian elements are considered negligible; thus, under the assumption of an ideal $180^{\circ}$ rotation for all spins, no coherence transfer occurs between coupled partners.

In previous studies an additional $180^{\circ}$ pulse was incorporated within a standard PRESS sequence and a MEGA-PRESS sequence to alleviate signal losses occurring as a consequence of chemical shift displacement of selected slices [10-12]. It was also suggested that this modification of PRESS, initially introduced for J-difference editing experiments, could be of value for any PRESS-based experiment, to reduce signal losses due to chemical shift displacement [11]. The results of this study indicate that it is necessary to assess the effects of broadband $180^{\circ}$ pulses on the spin evolution of strongly coupled systems, since large effects on the lineshape could occur at certain values of interpulse delays. In the work of Cunningham et al. [25], a train of $180^{\circ}$ pulses was also inserted within a PRESS sequence in order to minimize J-dephasing of the coupled resonances of citrate. The physical mechanism in this case is presumed to be a quenching of the J-modulation induced by a train of closely spaced refocusing pulses as discussed initially by Allerhand [26]. 
The physical mechanism underlying the phenomenon observed in the current study is the same mechanism that generates oscillations at constant TE as the timing of the first echo is varied [13] in the approach referred to as S-PRESS [14, 27] or constant TE PRESS difference spectroscopy [2] or difference spectroscopy using PRESS asymmetry [16]. Thus the additional $180^{\circ}$ pulse in gPRESS could provide a means for metabolite optimization and spectral editing in a single-shot fashion or as a difference spectroscopy method. Table 1 in the work of Snyder et al. [16] indicates that potential candidates for a spectral editing method that exploits this mechanism are the aspartate, glutamate, myo-inositol, $\mathrm{N}$-acetylaspartate, taurine, $\mathrm{N}$-acetylaspartylglutamate as well as citrate.

The 'perfect echo' was one of the first approaches employed for modifying the response of coupled spin systems within a double spin echo sequence. Interestingly, this concept has recently attracted the attention of the high resolution NMR community, with application to diffusion measurements [28] and it has been revisited by Aguilar et al. for the extension to an arbitrary spin system [29]. The 'perfect echo' approach, or J-refocusing method, has also found applications for in vivo MRS at high fields [30] with the aim of minimizing the signal dephasing (due to J-modulation), which at high fields becomes substantial even at short echo times.

All the aforementioned methods have one characteristic in common: they all share the notion that one or more pulses are incorporated within the PRESS localization sequence. The results of the current work indicate that caution must be taken when inserting a broadband $180^{\circ}$ in pulse sequences, due to its effect on the signal response of strongly coupled spin systems. Furthermore, the current work also indicates that the broadband $180^{\circ}$ pulse could be considered as an editing pulse, for selectively modifying the spectral shape of strongly coupled spin resonances.

\section{Acknowledgments}

GG gratefully acknowledges the generous support of Rennes Métropole. 


\section{Appendix}

The analytic solution of the transverse magnetization of an AB spin system $(\delta$, chemical shift difference and J, spin-spin coupling constant) under gPRESS excitation $\left(90^{\circ}{ }_{\mathrm{x}}-\left[\mathrm{TE}_{1} / 2\right]-180^{\circ}{ }_{\mathrm{y}}\right.$ $\left.-\left[\mathrm{TE}_{1} / 2\right]-180_{\mathrm{y}}^{\circ}-\left[\mathrm{TE}_{2} / 2\right]-180_{\mathrm{y}}^{\circ}-\left[\mathrm{TE}_{2} / 2\right]-\mathrm{Acq}\right)$ was obtained by use of the density matrix formalism.

The general solution was found of the form $S(t)=\Sigma A_{i} \cos \left[(d-b) t+\Psi_{i}\right]+B_{i} \cos \left[(d-c) t+\Phi_{i}\right]$. The solution consisted of 6 cosine terms for the 'inner lines', resonating at frequencies of $\pm(d-b)$ $\equiv \pm \beta$, and 6 cosine terms for the 'outer lines', resonating at frequencies of $\pm(d-c) \equiv \pm \Gamma$, where $d=J / 4, b=(-J / 4+\Omega / 2), c=(-J / 4-\Omega / 2)$, and $\Omega=\left(J^{2}+\delta^{2}\right)^{1 / 2}$. The frequencies $\beta$ and $\Gamma$ are the fundamental transition frequencies for the $\mathrm{AB}$ system. In Table $1, p=\cos (\theta / 2)$ and $q=\sin (\theta / 2)$, where $\theta=\arctan (J / \delta)$. Spectra were generated by Fourier transform over $t$ of $S(t)$. The Fourier transform yielded resonance peaks at $\pm \beta$ and $\pm \Gamma$, that correspond to the 'inner' and 'outer' lines of the $\mathrm{AB}$ quartet, respectively. Each line has a phase factor dependent on the interpulse delays and an amplitude that depends on $\mathrm{J}$ and $\delta$. The solution $S(t)$ is expressed succinctly in tabular form (Table 1 and 2). 


\section{References}

[1] P.A. Bottomley, Spatial localization in NMR spectroscopy in vivo, Ann. NY Acad. Sci. 508 (1987) 333-348.

[2] A. Yahya, Metabolite detection by proton magnetic resonance spectroscopy using PRESS, Prog. Nucl. Magn. Reson. Spectrosc. 55 (2009) 183-198.

[3] K. Takegoshi, K. Ogura, A. Hikichi, A perfect spin echo in a weakly homonuclear Jcoupled two spin 1/2 system, J. Magn. Reson. 84 (1989) 611-615.

[4] P.C.M. van Zijl, C. Moonen, M. von Kienlin, Homonuclear J refocusing in spin echo spectroscopy, J. Magn. Reson. 89 (1990) 28-40.

[5] R.V. Mulkern, J.L. Bowers, S. Peled, R.A. Kraft, D.S. Williamson, Citrate signal enhancement with a homonuclear J-refocusing modification to double-echo PRESS sequences, Magn. Reson. Med. 36 (1996) 775-80.

[6] A.H. Trabesinger, D.C. Mueller, P. Boesiger, Single-quantum coherence filter for strongly coupled spin systems for localized [1]H NMR spectroscopy, J. Magn. Reson. 145 (2000) 237-245.

[7] A.H. Trabesinger, O.M. Weber, C.O. Duc, P. Boesiger, Detection of glutathione in the human brain in vivo by means of double quantum coherence filtering, Magn. Reson. Med. 42 (1999) 283-289.

[8] J.R. Keltner, L.L. Wald, B.D. Frederick, P.F. Renshaw, In vivo detection of GABA in human brain using a localized double-quantum filter technique, Magn. Reson. Med. 37 (1997) 366-371.

[9] R.B. Thompson, P.S. Allen, A new multiple quantum filter design procedure for use on strongly coupled spin systems found in vivo: its application to glutamate, Magn. Reson. Med. 39 (1998) 762-771.

[10] S. Sison, M.H. Buonocore, R.J. Maddock, in: Proc. 15th Intl. Soc. Mag. Reson. Med., Berlin, Germany, (2007) 2932.

[11] L.G. Kaiser, K. Young, G.B. Matson, Elimination of spatial interference in PRESSlocalized editing spectroscopy Magn. Reson. Med. 58 (2007) 813-818.

[12] L.G. Kaiser, K. Young, D.J. Meyerhoff, S.G. Mueller, G.B. Matson, A detailed analysis of localized J-difference GABA editing: theoretical and experimental study at 4 T, NMR Biomed. 21 (2008) 22-32. 
[13] G. Gambarota, M. van der Graaf, D. Klomp, R.V. Mulkern, A. Heerschap, Echo-time independent signal modulations using PRESS sequences: a new approach to spectral editing of strongly coupled AB spin systems, J. Magn. Reson. 177 (2005) 299-306.

[14] T. Lange, A.H. Trabesinger, R.F. Schulte, U. Dydak, P. Boesiger, Prostate spectroscopy at 3 Tesla using two-dimensional S-PRESS, Magn. Reson. Med. 56 (2006) 1220-1228.

[15] N. Kickler, G. Gambarota, R. Mekle, R. Gruetter, R. Mulkern, Echo-time independent signal modulations for strongly coupled systems in triple echo localization schemes: an extension of S-PRESS editing, J. Magn. Reson. 203 (2010) 108-112.

[16] J. Snyder, R.B. Thompson, A.H. Wilman, Difference spectroscopy using PRESS asymmetry: application to glutamate, glutamine, and myo-inositol, NMR Biomed. 23 (2010) 41-47.

[17] M. van der Graaf, G.J. Jager, A. Heerschap, Removal of the outer lines of the citrate multiplet in proton magnetic resonance spectra of the prostatic gland by accurate timing of a point-resolved spectroscopy pulse sequence, MAGMA 5 (1997) 65-69.

[18] A.H. Wilman, P.S. Allen, The response of the strongly coupled AB system of citrate to typical 1H MRS localization sequences, J. Magn. Reson. B 107 (1995) 25-33.

[19] T.W. Scheenen, G. Gambarota, E. Weiland, D.W. Klomp, J.J. Fütterer, J.O. Barentsz, A. Heerschap, Optimal timing for in vivo $1 \mathrm{H}-\mathrm{MR}$ spectroscopic imaging of the human prostate at 3T, Magn. Reson. Med. 53 (2005) 1268-1274.

[20] K. Straubinger, F. Schick, O. Lutz, Computer-algebra calculations and measurements on AB spin systems for double-spin-echo sequences, MAGMA 3 (1995) 109-118.

[21] D. ter Haar, Theory and applications of the density matrix, Rep. Prog. Phys. 24 (1961) 304-362.

[22] U. Fano, Description of states in quantum mechanics by density matrix and operator techniques, Rev. Mod. Phys. 29 (1957) 74-93.

[23] R.V. Mulkern, J. Bowers, Density matrix calculations of AB spectra from multiple sequences: quantum mechanics meets in vivo spectroscopy, Concepts in Magnetic Resonance 6 (1994) 1-23.

[24] T.W. Scheenen, S.W. Heijmink, S.A. Roell, C.A. Hulsbergen-Van de Kaa, B.C. Knipscheer, J.A. Witjes, J.O. Barentsz, A. Heerschap, Three-dimensional proton MR spectroscopy of human prostate at $3 \mathrm{~T}$ without endorectal coil: feasibility, Radiology 254 (2007) 507-516. 
[25] C.H. Cunningham, D.B. Vigneron, M. Marjanska, A.P. Chen, D. Xu, R.E. Hurd, J. Kurhanewicz, M. Garwood, J.M. Pauly, Sequence design for magnetic resonance spectroscopic imaging of prostate cancer at 3 T, Magn. Reson. Med. 53 (2005) 10331039.

[26] A. Allerhand, Analysis of Carr-Purcell spin-echo NMR experiments on multiple-spin systems. I. The effect of homonuclear coupling, J. Chem. Phys. 44 (1966) 1-9.

[27] G. Gambarota, M. van der Graaf, D. Klomp, J. van Asten, A. Heerschap, S-PRESS: a novel approach to difference spectroscopy editing, In: Proc. 12th Intl. Soc. Mag. Reson. Med., Kyoto, Japan, (2004) 307.

[28] A.M. Torres, G. Zheng, W.S. Price, J-compensated PGSE: an improved NMR diffusion experiment with fewer phase distortions, Magn. Reson. Chem. 48 (2010) 129-133.

[29] J.A. Aguilar, M. Nilsson, G. Bodenhausen, G.A. Morris, Spin echo NMR spectra without J modulation, Chem. Commun. 48 (2012) 811-813.

[30] J.W. Pan, G.F. Mason, G.M. Pohost, H.P. Hetherington, Spectroscopic imaging of human brain glutamate by water-suppressed J-refocused coherence transfer at $4.1 \mathrm{~T}$, Magn. Reson. Med. 36 (1996) 7-12. 
Table 1. The amplitude and phase of the 6 cosine terms that generate the signal of the 'outer lines', at frequencies of $\pm(d-c) \equiv \pm \Gamma$, following gPRESS excitation $\left(90_{\mathrm{x}}-\mathrm{TE}_{1} / 2-180_{\mathrm{y}}-\right.$ $\mathrm{TE}_{1} / 2-180_{\mathrm{y}}-\mathrm{TE}_{2} / 2-180_{\mathrm{y}}-\mathrm{TE}_{2} / 2-\mathrm{Acq}$ ) of an $\mathrm{AB}$ spin system (see appendix for details).

\section{OUTER LINES}

\begin{tabular}{cc}
\hline Amplitude $(\mathrm{B})$ & Phase $(\Phi)$ \\
\hline $4 \mathrm{p}^{2} \mathrm{q}^{2}\left(\mathrm{p}^{2}-\mathrm{q}^{2}\right)^{2}$ & $\beta\left(\mathrm{TE}_{1}+\mathrm{TE}_{1} / 2\right)+\Gamma\left(\mathrm{TE}_{1} / 2\right)$ \\
\hline$\left(\mathrm{p}^{2}-\mathrm{q}^{2}\right)^{4}+2 \mathrm{pq}(\mathrm{p}-\mathrm{q})^{2}\left(\mathrm{p}^{2}-\mathrm{q}^{2}\right)^{2}$ & $\beta\left(\mathrm{TE}_{1} / 2+\mathrm{TE}_{2} / 2\right)+\Gamma\left(\mathrm{TE}_{1} / 2+\mathrm{TE}_{2} / 2\right)$ \\
\hline$-2 \mathrm{pq}(\mathrm{p}-\mathrm{q})^{2}\left(\mathrm{p}^{2}-\mathrm{q}^{2}\right)^{2}$ & $\Gamma\left(\mathrm{TE}_{1}+\mathrm{TE}_{1} / 2\right)+\beta \mathrm{TE}_{1} / 2$ \\
\hline$-4 \mathrm{p}^{2} \mathrm{q}^{2}\left(\mathrm{p}^{2}-\mathrm{q}^{2}\right)^{2}$ & $\beta\left(\mathrm{TE}_{1}\right)+\Gamma\left(\mathrm{TE}_{2}\right)$ \\
\hline $4 \mathrm{p}^{2} \mathrm{q}^{2}\left(\mathrm{p}^{2}-\mathrm{q}^{2}\right)^{2}-2 \mathrm{pq}(\mathrm{p}-\mathrm{q})^{2}\left(\mathrm{p}^{2}-\mathrm{q}^{2}\right)^{2}$ & $\beta\left(\mathrm{TE}_{1} / 2\right)+\Gamma\left(\mathrm{TE}_{1} / 2+\mathrm{TE}_{2}\right)$ \\
\hline$-8 \mathrm{p}^{3} \mathrm{q}^{3}(\mathrm{p}-\mathrm{q})^{2}$ & $\Gamma\left(\mathrm{TE}_{1}+\mathrm{TE}_{2}\right)$ \\
\hline
\end{tabular}


Table 2. The amplitude and phase of the 6 cosine terms that generate the signal of the inner lines', at frequencies of $\pm(d-b) \equiv \pm \beta$, following gPRESS excitation of an AB spin system (see appendix for details).

\section{INNER LINES}

\begin{tabular}{cc}
\hline Amplitude $(\mathrm{A})$ & Phase $(\Psi)$ \\
\hline $2 \mathrm{pq}(\mathrm{p}+\mathrm{q})^{2}\left(\mathrm{p}^{2}-\mathrm{q}^{2}\right)^{2}$ & $\beta\left(\mathrm{TE}_{1}+\mathrm{TE}_{1} / 2\right)+\Gamma\left(\mathrm{TE}_{1} / 2\right)$ \\
\hline$-2 \mathrm{pq}(\mathrm{p}+\mathrm{q})^{2}\left(\mathrm{p}^{2}-\mathrm{q}^{2}\right)^{2}+\left(\mathrm{p}^{2}-\mathrm{q}^{2}\right)^{4}$ & $\beta\left(\mathrm{TE}_{1} / 2+\mathrm{TE}_{2} / 2\right)+\Gamma\left(\mathrm{TE}_{1} / 2+\mathrm{TE}_{2} / 2\right)$ \\
\hline $4 \mathrm{p}^{2} \mathrm{q}^{2}\left(\mathrm{p}^{2}-\mathrm{q}^{2}\right)^{2}$ & $\Gamma\left(\mathrm{TE}_{1}+\mathrm{TE}_{1} / 2\right)+\beta \mathrm{TE}_{1} / 2$ \\
\hline$-4 \mathrm{p}^{2} \mathrm{q}^{2}\left(\mathrm{p}^{2}-\mathrm{q}^{2}\right)^{2}$ & $\beta\left(\mathrm{TE}_{2}\right)+\Gamma\left(\mathrm{TE}_{1}\right)$ \\
\hline $2 \mathrm{pq}(\mathrm{p}+\mathrm{q})^{2}\left(\mathrm{p}^{2}-\mathrm{q}^{2}\right)^{2}+4 \mathrm{p}^{2} \mathrm{q}^{2}\left(\mathrm{p}^{2}-\mathrm{q}^{2}\right)^{2}$ & $\beta\left(\mathrm{TE}_{1} / 2+\mathrm{TE}_{2}\right)+\Gamma\left(\mathrm{TE}_{1} / 2\right)$ \\
\hline $8 \mathrm{p}^{3} \mathrm{q}^{3}(\mathrm{p}+\mathrm{q})^{2}$ & \\
\end{tabular}


Figure 1. Simulations from density matrix theory of the citrate signal intensity at $11.7 \mathrm{~T}$. The signal difference between the double spin echo sequence without (PRESS sequence) and with (gPRESS sequence) the additional $180^{\circ}$ pulse is shown. Large differences for TE in the range of $60-80 \mathrm{~ms}, 120-140 \mathrm{~ms}$ and $180-200 \mathrm{~ms}$ are noticeable. Differences in signal reach values up to 1 (as evident for TE of $200 \mathrm{~ms}$ ). As the total signal intensity of the AB spin system of citrate has been normalized to the value of 2, this signal difference corresponds to $50 \%$ change. (For the sake of figure clarity, impossible combinations of $\mathrm{TE}_{1}$ and $\mathrm{TE}$ (i.e., such as $\mathrm{TE}<\mathrm{TE}_{1}$ ) have been kept in the graph).

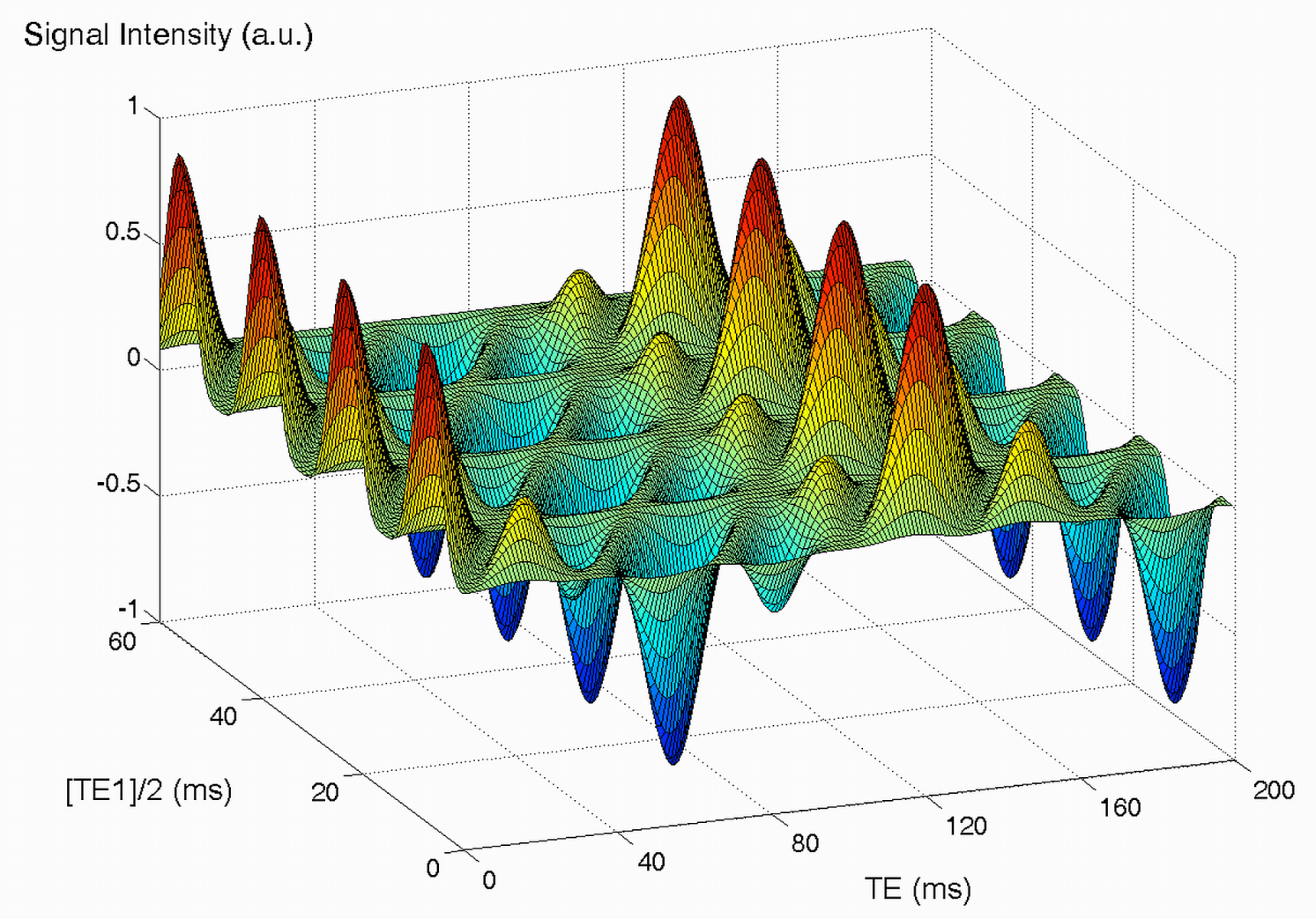


Figure 2. Simulated proton MR spectra of the citrate resonances at $11.7 \mathrm{~T}$ for the double spin echo sequence (PRESS sequence) and for the double spin echo sequence with the additional $180^{\circ}$ pulse (gPRESS sequence). The sequence delays (total $\mathrm{TE}=132 \mathrm{~ms}$ and $\mathrm{TE}_{1}=20 \mathrm{~ms}$ ) are chosen to illustrate here the large effect of the $180^{\circ}$ pulse on the spectral shape and signal intensity of the citrate resonances. The same vertical scale is used for both spectra.

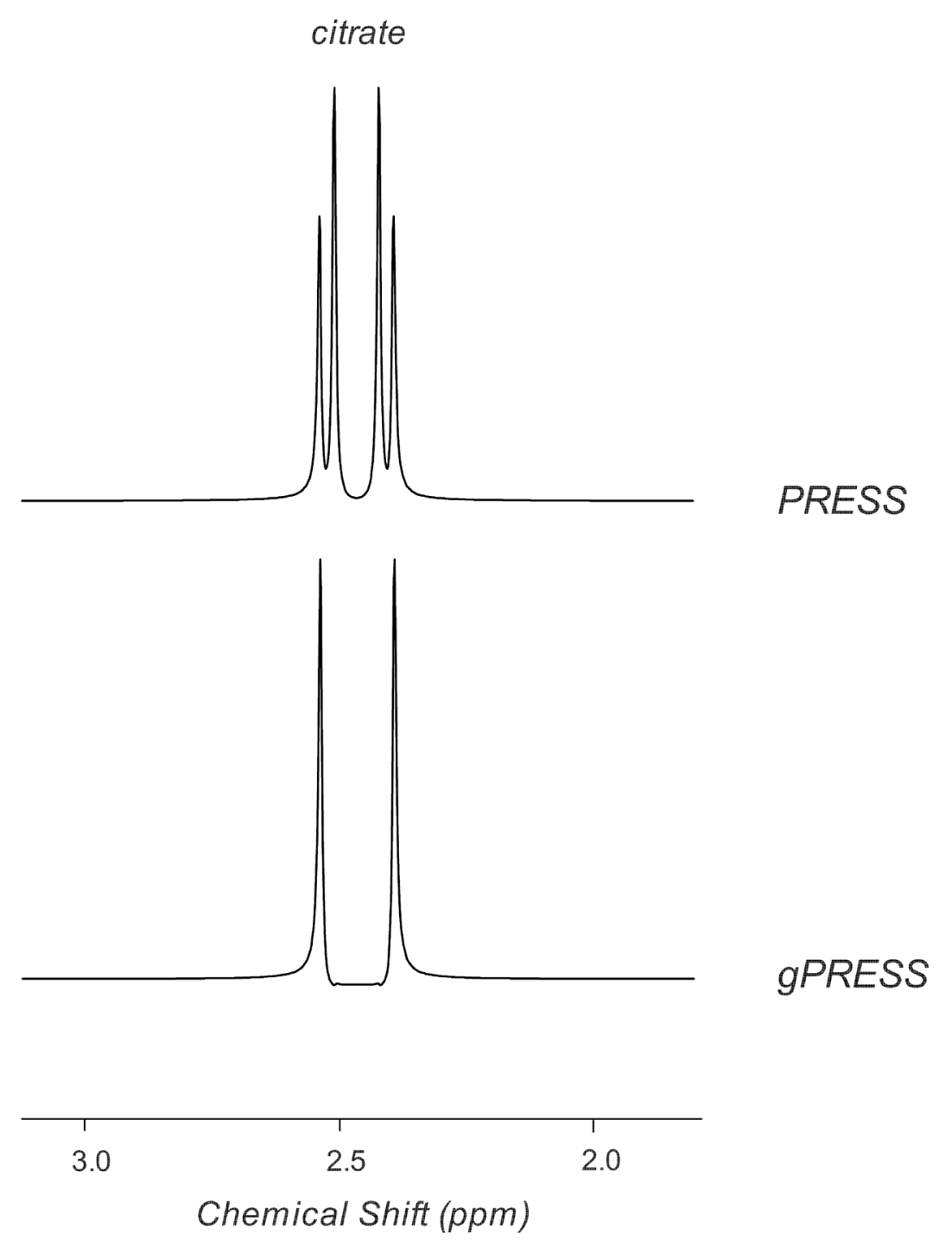


Figure 3. Spectra acquired at $11.7 \mathrm{~T}$ from a phantom containing citrate, lactate and glycine. The additional $180^{\circ}$ pulse in the double spin echo sequence has a large effect on the citrate resonances, but no effect on the glycine (singlet) and lactate (weakly coupled) resonances. The sequence timings are $\mathrm{TE}=132 \mathrm{~ms}$ and $\mathrm{TE}_{1}=20 \mathrm{~ms}$. The spectral shape of the citrate resonances agrees well with the simulated spectra shown in Figure 2.

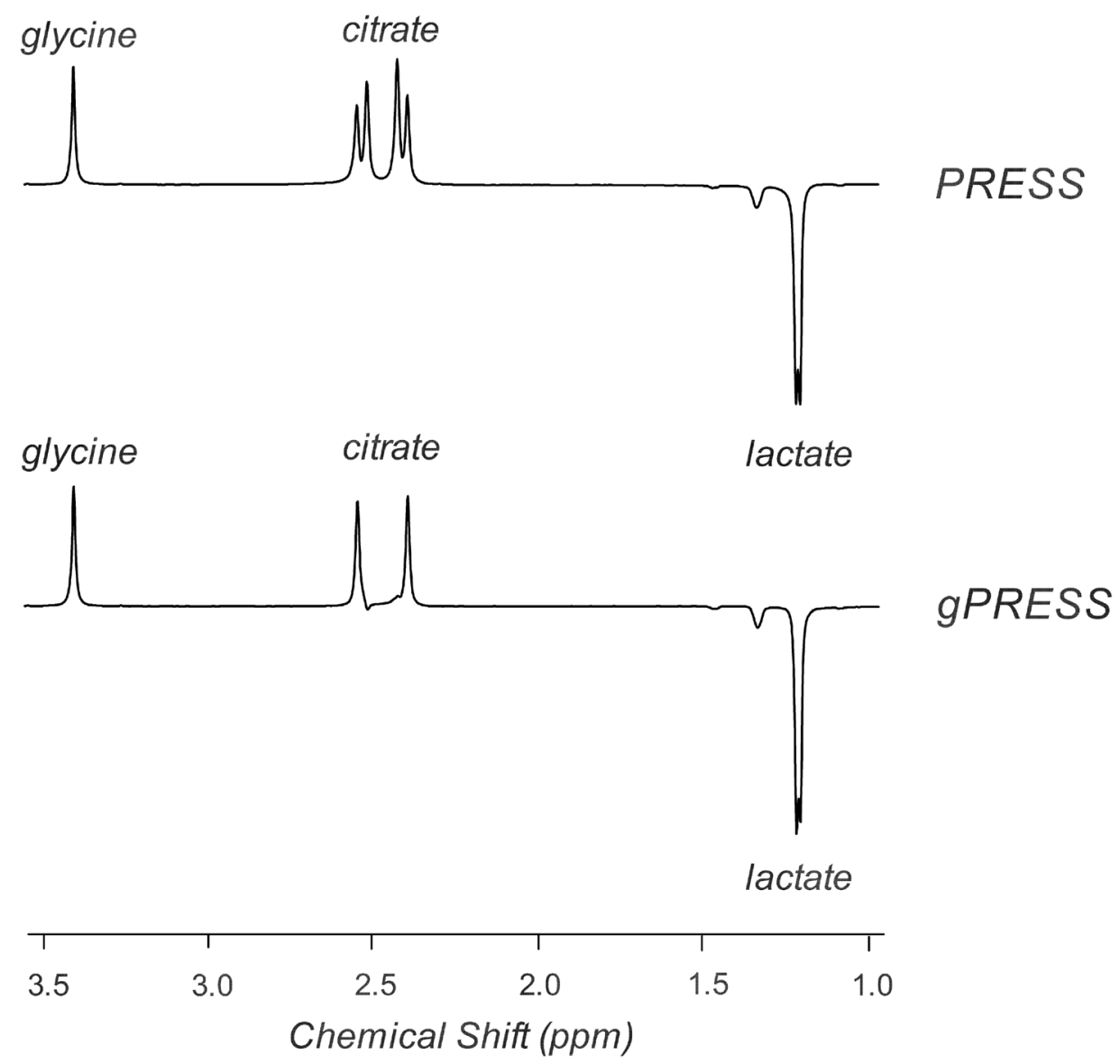


Figure 4. Simulated proton MR spectra of the citrate resonances at $3 \mathrm{~T}$ for the double spin echo sequence with the additional $180^{\circ}$ pulse (gPRESS sequence) and for the double spin echo sequence (PRESS sequence). The gPRESS sequence yields a favourable spectral shape at $\mathrm{TE}=67 \mathrm{~ms}\left(\mathrm{TE}_{1}=34 \mathrm{~ms}\right)$. The spectrum of PRESS with $\mathrm{TE}=145 \mathrm{~ms}$ (and $\mathrm{TE}_{1}=50 \mathrm{~ms}$, commonly used timings for citrate quantitation at 3T [24]) is also shown. Furthermore, the citrate signal for PRESS at TE $=67 \mathrm{~ms}$ and gPRESS at $145 \mathrm{~ms}$ is shown here, for comparison purposes. No T2 relaxation effects are taken into account in the simulated spectra and the same vertical scale is used for all spectra.

gPRESS

$T E=67 \mathrm{~ms}$

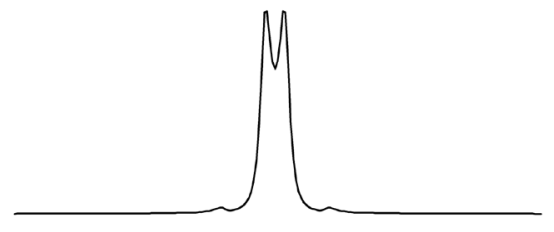

PRESS

$T E=67 \mathrm{~ms}$
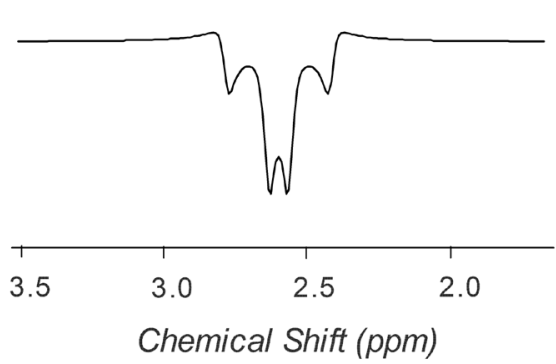

PRESS

$T E=145 \mathrm{~ms}$

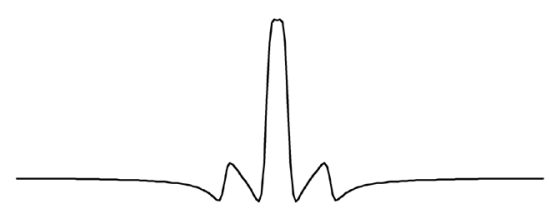

gPRESS

$T E=145 \mathrm{~ms}$

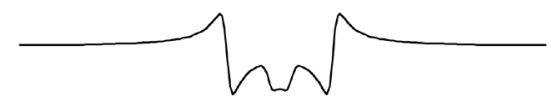

\begin{tabular}{llll}
\hline 1 & 1 & 2.5 & 2.0 \\
3.5 & 3.0 & 2.5 & \\
& \multicolumn{4}{c}{ Chemical Shift (ppm) }
\end{tabular}


Figure 5. Proton MR spectra of the citrate resonances at $11.7 \mathrm{~T}$ for PRESS (continuous line) and gPRESS (dashed line) obtained by the analytic solution, with $\mathrm{TE}=132 \mathrm{~ms}$ and $\mathrm{TE}_{1}=20$ ms. These spectra are in excellent agreement with the spectra obtained from density matrix simulations (Figure 2).

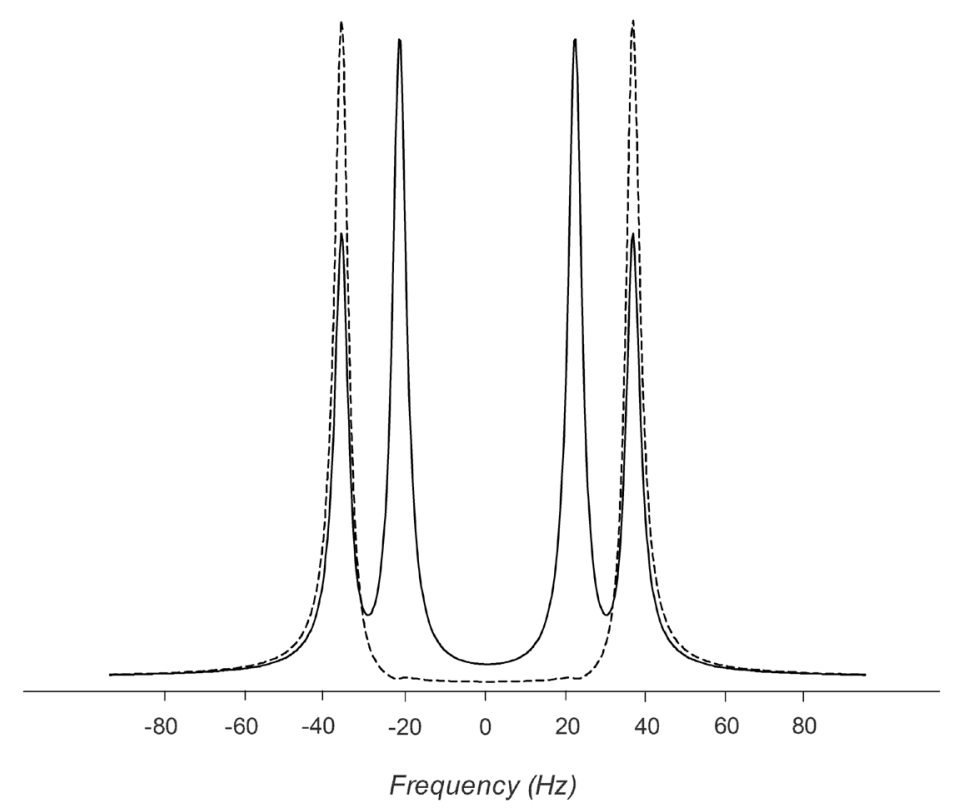

